\title{
Clinical relevance and utility of cetuximab-related changes in magnesium and calcium serum levels
}

Sebastian Stintzing ${ }^{a}$, Doris Fischhaber ${ }^{a}$, Charlotte Mook ${ }^{a}$, Dominik P. Modest ${ }^{a}$, Clemens Giessen ${ }^{a}$, Christoph Schulz ${ }^{a}$, Michael Haas ${ }^{a}$, Stefan Boeck ${ }^{a}$, Marlies Michl ${ }^{a}$, Joachim Stemmler ${ }^{a}$, Rüdiger P. Laubender ${ }^{b}$ and Volker Heinemann ${ }^{a}$

\begin{abstract}
Hypomagnesemia and hypocalcemia are common adverse events during cetuximab treatment. The influence of the chemotherapeutic combination on serum levels is unknown and the predictive value is currently under discussion. This analysis investigated 79 patients who had received cetuximab for at least 6 weeks in the day clinic of the Comprehensive Cancer Center, University of Munich. Calcium and magnesium serum levels were analyzed weekly; tumor response and adverse events were followed. Thirty-eight patients had metastatic colorectal cancer (mCRC) and the predictive value of hypomagnesemia was tested in these patients. During therapy, calcium serum levels decreased to about $97 \%$ of the baseline levels and were maintained for the duration of treatment. Magnesium levels showed a significant time-dependent decrease. Serum levels of magnesium were lower when cetuximab was combined with a platinum derivative. After a treatment duration of 12 weeks, magnesium levels decreased to $70 \%$ in platinum-treated patients, whereas they decreased to only $90 \%$ of baseline in patients who did not receive platinum therapy. In patients treated for $\mathrm{MCRC}$, a decrease of serum magnesium below
\end{abstract}

\section{Introduction}

Treatment with antibodies directed against the epidermal growth factor receptor (EGFR) has improved antitumor treatment efficacy in several entities. Next to the treatment of mCRC [1-3], the anti-EGFR-antibody cetuximab is approved for the treatment of squamous cell cancer of the head and neck [4], and was also evaluated in other malignancies such as lung cancer and gastric cancer [5,6]. The occurrence of hypomagnesemia as a side effect of cetuximab treatment has been observed within the first trials using cetuximab [7] and appears to be a class effect of anti-EGFR antibodies [8]. The mechanism of hypomagnesemia in anti-EGFR treated patients has been explained by the link between TRMP6 (transient receptor potential member 6) and EGFR signaling. Magnesium is absorbed in the gut and reabsorbed in the ascending loop of Henle by TRMP6 [9], an ion channel. TRMP6 activation is mediated by EGFR signaling [10].

Next to acneiform exanthema [11], hypomagnesemia [12] has also been described as a possible clinical predictor for outcome. However, conflicting data were reported in
$95 \%$ of the baseline levels 14 days after initiating treatment separated patients significantly in terms of survival times. Magnesium levels decrease in a time-dependent manner during cetuximab therapy. As hypomagnesemia was more prominent in patients receiving platinum agents, magnesium measurements may be advised in these patients. In mCRC patients treated with cetuximab, day-14 magnesium serum levels correlated with treatment efficacy. Anti-Cancer Drugs 24:969-974 @ 2013 Wolters Kluwer Health | Lippincott Williams \& Wilkins.

Anti-Cancer Drugs 2013, 24:969-974

Keywords: cetuximab, colon cancer, hypomagnesemia, magnesium, oxaliplatin

aDepartment of Internal Medicine III and Comprehensive Cancer Center, Klinikum Grosshadern and 'Institute of Medical Informatics, Biometry and Epidemiology, University of Munich, Munich, Germany

Correspondence to Sebastian Stintzing, Department of Internal Medicine III and Comprehensive Cancer Center, Klinikum Grosshadern, University of Munich, Marchioninistrasse 15, 81377 Munich, Germany

Tel: +49897095 2208; fax: +49897095 5256;

e-mail: sebastian.stintzing@med.uni-muenchen.de

Received 12 March 2013 Revised form accepted 10 June 2013

a recent report, indicating that hypomagnesemia induced by cetuximab monotherapy was associated with inferior survival [13]. Therefore, the predictive value of magnesium and calcium decrease is currently under discussion. The effect of the chemotherapeutic drugs used in combination with cetuximab on the occurrence and frequency of hypomagnesemia has not been elucidated. Furthermore, risk factors defining patients who are more likely to develop hypomagnesemia have not yet been identified.

The present analysis aimed to define the course of magnesium and calcium serum levels during cetuximab exposure and searched for risk factors predicting the development of hypomagnesemia and hypocalcemia. In this context, specific focus was on the effect of different chemotherapy backbones on the development of hypomagnesemia and hypocalcemia.

\section{Patients and methods}

Patients receiving cetuximab for cancer treatment in the day clinic of the Comprehensive Cancer Center LMU, Klinikum der Universität, University of Munich, were 
investigated. In this retrospective analysis, patients treated from 2009 to 2011 were included. Cetuximab was administered according to the manufacturer's instructions. After a loading dose of $400 \mathrm{mg} / \mathrm{m}^{2}$ (120 min intravenously), cetuximab was administered weekly at a dose of $250 \mathrm{mg} / \mathrm{m}^{2}$ (90-30 min intravenously). Concomitant medication with dimethindene (4 mg intravenously) and dexamethasone (4 mg intravenously) was administered at all treatment cycles. In addition, patients receiving oxaliplatin for colorectal cancer treatment were premedicated with calcium gluconate ( $1 \mathrm{~g}$ intravenously) and magnesium sulfate ( $1 \mathrm{~g}$ intravenously) as prophylaxis for peripheral neuropathy. In evaluable patients, duration of cetuximab exposure of at least 6 weeks was required. To limit the number of parameters included in this analysis and possibly weakening our conclusions, patients treated with panitumumab were excluded from this study.

\section{Clinical parameters}

Before each treatment, standard laboratory tests, including serum creatinine, glomerular filtration rate (GFR) calculated using the Cockroft-Gault formula, magnesium, and calcium, were carried out. Toxicity was evaluated by a standardized toxicity form, including acneiform exanthema, skin and nail changes, and diarrhea as cetuximab-specific parameters. Grading was performed according to NCI CTC AE version 3.0 (National Institutes of Health, Bethesda, Maryland, USA). Also, basic patient characteristics (age, sex, tumor type, KRAS mutational status, chemotherapeutic regimen, and treatment line) were determined. Treatment efficacy was defined by best overall response (RECIST 1.0) and survival times (progression-free survival and overall survival). The Ethics Committee of the University of Munich (Grosshadern) approved the study design (\#081-13).

\section{Statistical analyses}

Logistic regression exploration was carried out for univariate and multivariate analyses to evaluate the influence of baseline characteristics (age, sex, KRAS status, serum creatinine, GFR, tumor type, chemotherapeutic drugs) on changes in the calcium and magnesium serum levels. To define cut-off levels for decrease in magnesium, a receiver-operator characteristics (ROG) curve was constructed and the cut-off was set where the sum of specificity and sensitivity reached its maximum. To calculate differences in response, Fisher's exact test was used. For differences in survival, Kaplan-Meier estimates and $\log$-rank $P$ were calculated. To evaluate the time-dependent manner of magnesium and calcium levels, analysis of variance testing for repeated measurements was carried out.

All statistical tests were two-sided and a $P$-value of less than 0.05 was considered statistically significant. R (version 2.11.1; R Foundation, Vienna, Austria) and
SPSS PASW 20.0 (SPSS Inc., Chicago, Illinois, USA) software were used for all statistical analyses.

\section{Results}

A total of 97 patients received cetuximab during the period from 2009 to 2011. Of these, 79 received cetuximab for a period of at least 6 weeks and therefore were included in this analysis (for baseline characteristics, Table 1a). For each parameter (magnesium and calcium), 1359 serum measurements were available for analysis. The toxicities of interest (hypomagnesemia, hypocalcemia, acneiform exanthema) were as presented in Table $1 \mathrm{~b}$ and c. In brief, a total of nine patients (11.4\%) developed hypomagnesemia reaching NCI CTC grade 3-4 and six patients (7.6\%) developed a grade 3-4 hypocalcemia. By plotting the mean magnesium and calcium levels over time in relation to the baseline measurements (Fig. 1), two differences could be observed. Calcium levels decreased nonsignificantly during the first 2 weeks to a level of about $95-97 \%$ of baseline and were maintained for the entire duration of treatment. Therefore, calcium levels did not seem to be a valuable parameter to correlate with treatment efficacy. In comparison, magnesium levels decreased significantly (Greenhouse-Geisser $P=0.03$ ) in a time-dependent manner without reaching a minimum. Furthermore, a difference could be observed between the magnesium levels in patients treated with any platinum derivative and cetuximab and those who received platinum-free chemotherapy. In patients treated with platinum derivatives, the decrease in magnesium was faster and reached lower levels (about $70 \%$ of baseline) than in patients not treated with a platinum derivative, in whom levels reached $85-90 \%$ of baseline after a treatment duration of 12 weeks. Using repeated-measures analysis of variance testing, the difference between platinum and nonplatinum patients in terms of the course of magnesium did not differ significantly (Greenhouse-Geisser $P=0.24$ ), but showed a trend towards lower levels in platinumtreated patients.

Using Kendall-tau- $\beta$ rank testing, the occurrence of acneiform exanthema correlated significantly with the decrease of serum magnesium levels (correlation coefficient $-0.21 ; P=0.037)$.

Univariate and multivariate analyses did not show any association with baseline characteristics and magnesium levels after 2 weeks of treatment (Table 2). With respect to calcium levels, the baseline serum creatinine level was found to be an independent factor for lower calcium levels in univariate [odds ratio $(\mathrm{OR})=0.61 ; P=0.03$ ] and multivariate $(\mathrm{OR}=0.37 ; \quad P=0.006)$ analyses (Table 3).

For the subgroup of patients with mCRC, representing the largest subgroup in our analysis, logistic regression analysis indicated that lower magnesium levels at day 14 


\begin{tabular}{|c|c|c|c|c|}
\hline & \multicolumn{3}{|c|}{$n(\%)$} & \multirow[b]{2}{*}{ Total $(n=79)$} \\
\hline & $\mathrm{mCRC}(n=38)$ & $\operatorname{SCCHN}(n=27)$ & Other $^{\mathrm{a}}(n=14)$ & \\
\hline \multicolumn{5}{|c|}{ (a) Baseline characteristics } \\
\hline Age (years) (range) & $63.3(35.3-84.2)$ & $62.4(46.4-86.2)$ & $68.7(27.9-84.0)$ & $62.2(27.9-86.2)$ \\
\hline \multicolumn{5}{|l|}{ Sex } \\
\hline Male & $30(78.9)$ & $21(77.8)$ & $9(64.3)$ & $60(75.9)$ \\
\hline Female & $8(21.1)$ & $6(22.2)$ & $5(35.7)$ & $19(24.1)$ \\
\hline \multicolumn{5}{|l|}{ KRAS status } \\
\hline Wild-type & $27(71.1)$ & $3(11.1)$ & $7(50.0)$ & $37(46.8)$ \\
\hline Mutated & 7 (18.4) & 0 & $1(7.1)$ & $8(10.1)$ \\
\hline Not known & $4(10.5)$ & $24(88.9)$ & $6(42.9)$ & $34(43.1)$ \\
\hline \multicolumn{5}{|l|}{ Combination partner } \\
\hline Platinum & $3(7.9)$ & $13(48.1)$ & $7(50.0)$ & $23(29.1)$ \\
\hline Nonplatinum & $35(92.1)$ & $14(51.9)$ & $7(50.0)$ & $56(70.9)$ \\
\hline \multicolumn{5}{|l|}{ Therapy line } \\
\hline First line & $14(36.8)$ & $15(55.6)$ & $5(35.7)$ & $34(43.0)$ \\
\hline Further line & $24(63.2)$ & $12(44.4)$ & $9(64.3)$ & $45(56.9)$ \\
\hline \multicolumn{5}{|c|}{ (b) Toxicity ( $\mathrm{NCl} \mathrm{CTC}$ AE version 3.0 ) by cancer type } \\
\hline \multicolumn{5}{|c|}{ Hypomagnesemia } \\
\hline Grade 0 & $21(55.3)$ & $13(48.1)$ & $6(42.9)$ & $40(50.6)$ \\
\hline Grade 1 & $11(28.9)$ & $6(22.2)$ & $3(21.4)$ & $20(25.3)$ \\
\hline Grade 2 & $2(5.3)$ & $6(22.2)$ & $2(14.3)$ & $10(12.7)$ \\
\hline Grade 3/4 & $4(10.5)$ & $2(7.4)$ & $3(21.4)$ & $9(11.4)$ \\
\hline \multicolumn{5}{|l|}{ Hypocalcemia } \\
\hline Grade 0 & $27(71.1)$ & $22(81.5)$ & $7(50.0)$ & $56(70.9)$ \\
\hline Grade 1 & $8(21.1)$ & $2(7.4)$ & $1(7.1)$ & $11(13.9)$ \\
\hline Grade 2 & $2(5.3)$ & $1(3.7)$ & $3(21.4)$ & $6(7.6)$ \\
\hline Grade 3/4 & $1(2.6)$ & $2(7.4)$ & $3(21.4)$ & $6(7.6)$ \\
\hline \multicolumn{5}{|l|}{ Acneiform exanthema } \\
\hline Grade 0-1 & $7(18.4)$ & $8(29.6)$ & $6(42.9)$ & $21(26.6)$ \\
\hline Grade 2-4 & $29(76.3)$ & $17(62.9)$ & $8(57.1)$ & $54(68.4)$ \\
\hline Unknown & $2(5.3)$ & $2(7.4)$ & 0 & $4(5.1)$ \\
\hline & Nonplatinum-containing regimen $(n=56)$ & Platinum-containing regimen $(n=23)$ & Total $(n=79)$ & \\
\hline \multicolumn{5}{|c|}{$\begin{array}{l}\text { (c) Toxicity ( } \mathrm{NCI} \mathrm{CTC} \text { AE version 3.0) by platinum-based chemotherapy } \\
\text { Hypomagnesemia }\end{array}$} \\
\hline Grade 0 & $33(58.9)$ & $7(30.4)$ & $40(50.6)$ & \\
\hline Grade 1 & $13(23.2)$ & $7(30.4)$ & $20(25.3)$ & \\
\hline Grade 2 & $6(10.7)$ & $4(17.4)$ & $10(12.7)$ & \\
\hline Grade 3/4 & $4(7.2)$ & $5(21.7)$ & $9(11.4)$ & \\
\hline \multicolumn{5}{|l|}{ Hypocalcemia } \\
\hline Grade 0 & $41(73.2)$ & $15(65.2)$ & $56(70.9)$ & \\
\hline Grade 1 & $11(19.6)$ & 0 & $11(13.9)$ & \\
\hline Grade 2 & $2(13.6)$ & $4(17.4)$ & $6(7.6)$ & \\
\hline Grade 3/4 & $2(13.6)$ & $4(17.4)$ & $6(7.6)$ & \\
\hline \multicolumn{5}{|l|}{ Acneiform exanthema } \\
\hline Grade 0-1 & $11(19.6)$ & $10(43.5)$ & $21(26.6)$ & \\
\hline Grade 2-4 & $42(75.0)$ & $12(52.2)$ & $54(68.4)$ & \\
\hline Unknown & $3(5.4)$ & $1(4.3)$ & $4(5.1)$ & \\
\hline
\end{tabular}

mCRC, metastatic colorectal cancer; SCCHN, squamous cell cancer of the head and neck.

${ }^{a}$ Other refers to: four undifferentiated adenoid tumors of the head and neck, three carcinomas of unknown primary (CUP) with adenoid differentiation, two gastric cancers, one of each: adenocarcinoma of the lung, breast cancer, prostate cancer.

were significantly associated with higher overall response rates (ORR) $(\mathrm{OR}=1.52 ; P=0.03)$. A similar trend was observed for disease control rate but did not reach significance $(\mathrm{OR}=1.34 ; P=0.08)$. Calcium was not associated with either ORR or disease control rate (Table 4).

On constructing ROC curves for response in mCRC patients, the area under the curve reached 0.625 and the sum of specificity and sensitivity reached its maximum at a decrease of magnesium on day 14 of cetuximab treatment to $95 \%$ of the baseline value.

Using this discrimination, patients with lower magnesium levels had a significantly longer progression-free survival (4.6 vs. 2.5 months; $\log$-rank $P=0.03$; hazard ratio 0.42 ) and a trend toward longer overall survival (20.0 vs. 4.4 months, $\log$-rank $P=0.12$; hazard ratio 0.45 ) (Fig. 2). ORR was doubled in patients with lower magnesium levels on day 14 , but the difference (33.3 vs. $66.7 \%$ ) did not reach the level of significance (Fisher's exact $P=0.053$ ).

\section{Discussion}

Hypomagnesemia has been noted as an adverse event already after the first trials investigating cetuximab $[7,14]$. Magnesium wasting is supposedly caused by an inhibitory effect of anti-EGFR agents on TRMP6, which is responsible for reabsorption of magnesium in the ascending limp of the loop of Henle [7,9,15]. In most patients, hypomagnesemia remains asymptomatic and does not lead to clinical symptoms such as muscle cramps, fatigue, or general weakness [14]. Therefore, 
magnesium serum levels have only been followed in a small fraction of patients participating in clinical trials $[1,4,8]$. Early changes in magnesium levels have

Fig. 1

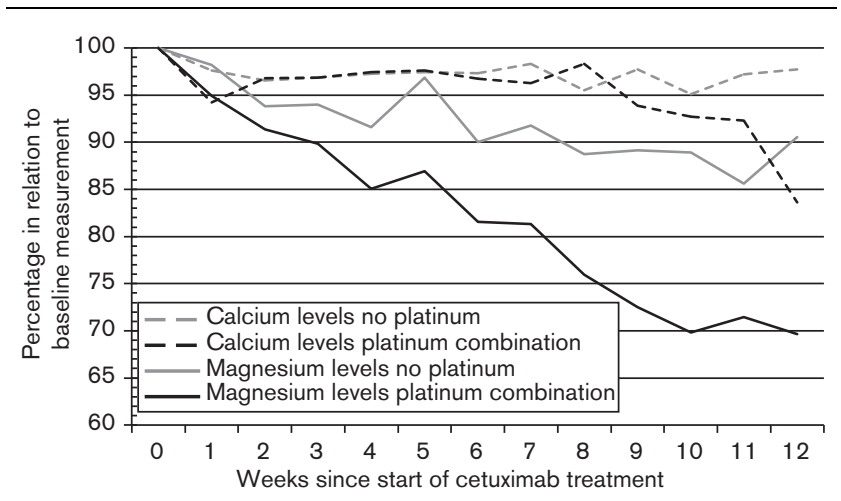

Course of calcium and magnesium during cetuximab treatment. been described recently as a surrogate parameter for treatment efficacy in the third-line treatment of mCRC [12] with irinotecan and cetuximab. Another study reported a negative prognostic effect when cetuximab was provided as monotherapy [13] in heavily pretreated patients.

Even the present analysis has some limitations because of its retrospective nature and possible selection bias as patients treated for less than 6 weeks were excluded; this indicates a time-dependent, significant decrease in magnesium serum levels in patients treated with cetuximab. Using baseline characteristics for a logistic regression analysis, no risk factor could be established for the decrease in magnesium (Table 2). Risk factors, therefore, may be found in genetic analyses, but to date, no known factor to predict hypomagnesemia has been established from clinical baseline parameters.

Hypocalcemia is a rare event in cetuximab-treated patients [16] and it is unclear whether lower calcium

Table 2 Univariate and multivariate logistic regression analysis of baseline factors to predict magnesium decrease on day 14

\begin{tabular}{|c|c|c|c|c|}
\hline & OR (univariate) $(95 \% \mathrm{Cl})$ & $P$ & OR (multivariate) $(95 \% \mathrm{Cl})$ & $P$ \\
\hline Age (continuous) (years) & $1.01(0.99-1.02)$ & 0.30 & $1.00(0.99-1.02)$ & 0.68 \\
\hline Sex (female) & $1.18(0.86-1.62)$ & 0.17 & $1.29(0.82-2.02)$ & 0.27 \\
\hline $\mathrm{SCCHN}$ (mCRC as standard) & $1.22(0.93-1.60)$ & 0.17 & $1.12(0.76-1.65)$ & 0.56 \\
\hline Other tumor (mCRC as standard) & $0.85(0.58-1.25)$ & 0.41 & $0.89(0.57-1.41)$ & 0.63 \\
\hline Platinum & $0.93(0.70-1.23)$ & 0.60 & $0.85(0.58-1.25)$ & 0.42 \\
\hline Creatinine (log) (continuous) & $1.09(0.68-1.75)$ & 0.72 & $0.60(0.27-1.31)$ & 0.20 \\
\hline GFR (log) (continuous) & $0.44(0.13-1.47)$ & 0.19 & $0.31(0.06-1.64)$ & 0.17 \\
\hline KRAS mutation & $1.07(0.94-1.22)$ & 0.34 & $1.05(0.87-1.27)$ & 0.63 \\
\hline
\end{tabular}

$\mathrm{Cl}$, confidence interval; GFR, glomerular filtration rate; log, logarithmic values; mCRC, metastatic colorectal cancer; OR, odds ratio; $P$, logistic regression analysis $P$; $\mathrm{SCCHN}$, squamous cell cancer of the head and neck.

Table 3 Univariate and multivariate logistic regression analyses of baseline factors to predict calcium decrease on day 14

\begin{tabular}{|c|c|c|c|c|}
\hline & OR (univariate) $(95 \% \mathrm{Cl})$ & $P$ & OR (multivariate) $(95 \% \mathrm{Cl})$ & $P$ \\
\hline Age (continuous) (years) & $1.00(1.00-1.01)$ & 0.29 & $1.01(1.00-1.02)$ & 0.12 \\
\hline Sex (female) & $1.04(0.80-1.35)$ & 0.80 & $1.43(0.96-1.88)$ & 0.09 \\
\hline $\mathrm{SCCHN}$ (mCRC as standard) & $1.03(0.81-1.34)$ & 0.74 & $0.78(0.62-1.22)$ & 0.41 \\
\hline Other tumor $\mathrm{mCRC}$ as standard) & $0.86(0.63-1.17)$ & 0.33 & $0.78(0.57-1.08)$ & 0.14 \\
\hline Platinum & $0.97(0.76-1.24)$ & 0.81 & $1.02(0.75-1.37)$ & 0.93 \\
\hline Creatinine (log) (continuous) & $0.61(0.40-0.94)$ & $0.03^{\mathrm{a}}$ & $0.37(0.19-0.73)$ & 0.006 \\
\hline GFR (log) (continuous) & $2.66(0.82-8.62)$ & 0.11 & $0.85(0.19-3.90)$ & 0.83 \\
\hline KRAS mutation & $1.04(0.93-1.18)$ & 0.48 & $1.06(0.91-1.24)$ & 0.46 \\
\hline
\end{tabular}

$\mathrm{Cl}$, confidence interval; log, logarithmic values; $\mathrm{mCRC}$, metastatic colorectal cancer; OR, odds ratio; $P$, logistic regression analysis $P$; SCCHN, squamous cell cancer of the head and neck; GFR, glomerular filtration rate.

${ }^{a}$ Statistical significant data are given in bold.

Table 4 Correlation of changes of calcium and magnesium (baseline vs. day 14) with parameters of treatment efficacy

\begin{tabular}{|c|c|c|c|c|}
\hline & $\operatorname{mCRC}(n=38)(95 \% \mathrm{Cl})$ & $P$ & Database $(n=79)(95 \% \mathrm{Cl})$ & $P$ \\
\hline \multicolumn{5}{|l|}{ ORR } \\
\hline Magnesium (day 14) & $1.52(1.07-2.15)$ & $0.03^{\mathrm{a}}$ & $1.15(0.89-1.50)$ & 0.30 \\
\hline Calcium (day 14) & $0.95(0.68-1.32)$ & 0.74 & $0.88(0.71-1.11)$ & 0.30 \\
\hline \multicolumn{5}{|l|}{ DCR } \\
\hline Magnesium (day 14) & $1.34(0.98-1.84)$ & 0.08 & $0.94(0.74-1.20)$ & 0.64 \\
\hline Calcium (day 14) & $0.90(0.68-1.18)$ & 0.44 & $0.88(0.71-1.08)$ & 0.23 \\
\hline
\end{tabular}

$\mathrm{Cl}$, confidence interval; $\mathrm{DCR}$, disease control rate; $\mathrm{mCRC}$, metastatic colorectal cancer; ORR, overall response rate; $P$, Fisher's exact test $P$.

${ }^{\text {a }}$ Statistical significant data are given in bold. 

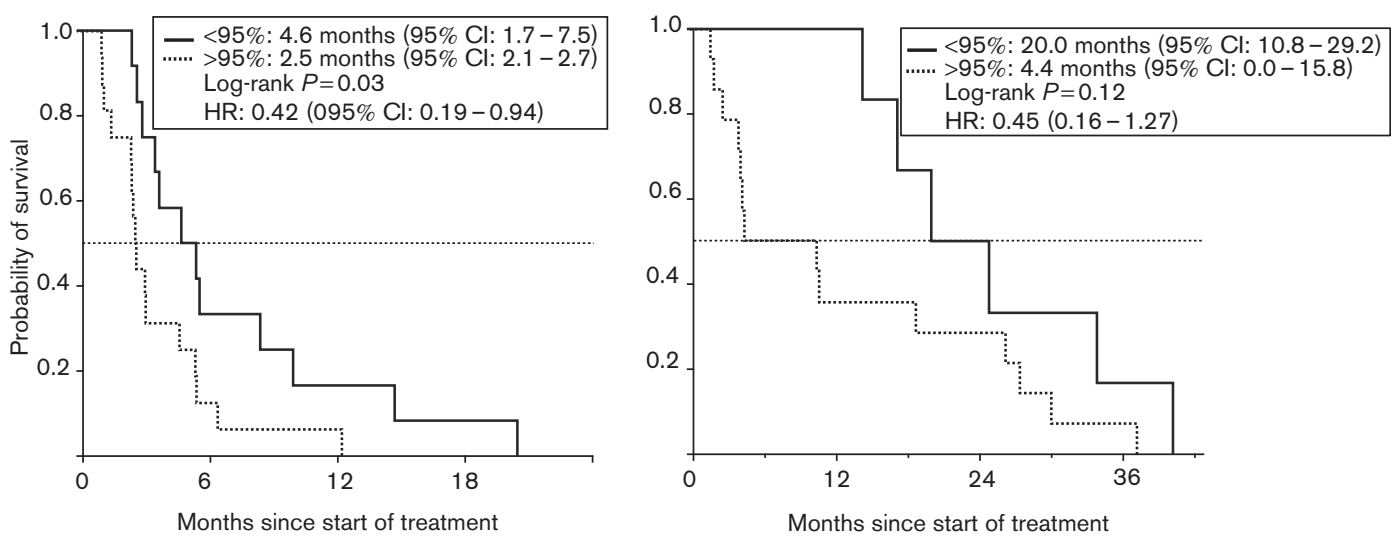

(a) Progression-free survival and (b) overall survival in patients with metastatic colorectal cancer; predictive value of magnesium decrease to day 14. $<95 \%$, magnesium serum levels $<95 \%$ of baseline; $>95 \%$, magnesium serum levels $>95 \%$ of baseline; $95 \% \mathrm{Cl}$, $95 \%$ confidence interval; HR, hazard ratio.

levels are because of changes induced by cetuximab or secondary to changes in the kidney [17]. The phenomenon of hypomagnesemic hypocalcemia is well known [18] and may therefore contribute to the hypocalcemia in cetuximab-treated patients. Hypocalcemia may further be associated with a preexisting impairment of renal function. In univariate and multivariate logistic regression analyses, baseline serum creatinine levels were found to be predictive for hypocalcemia. This indicates that a closer follow-up should be performed of patients with borderline serum creatinine levels for hypocalcemia when treated with cetuximab.

The incidence of grade 3-4 hypomagnesemia in this cohort was $11 \%$, which is somewhat higher than that reported before $(3-5 \%)$ [7,14]. This may be attributed to the study design as only patients who had received cetuximab for at least 6 weeks were included and because magnesium levels decrease in a time-dependent manner [19]. Hypomagnesemia of any grade could be detected in $49.6 \%$ of patients. This rate is higher than that reported by Petrelli et al. [8], but it is within the range of another report in which all-grade hypomagnesemia was observed in $56 \%$ of patients [16]. The discordant findings may indicate that the lack of routine magnesium determinations is likely to result in an underestimation of the frequency and severity of hypomagnesemia during cetuximab exposure.

The present analysis suggests that patients treated with cetuximab plus platinum derivatives show a faster decrease in magnesium levels that results in markedly lower serum concentrations of magnesium (about $70 \%$ of baseline) compared with patients receiving cetuximab with other combinations. This platinum-related difference in the pattern of magnesium kinetics has not been described before. Previous studies carried out in mCRC comparing oxaliplatin-based regimens with irinotecanbased regimen $[20,21]$ did not compare the frequency or the grade of hypomagnesemia. It may only be speculated that this was in part because of the insignificant effect of hypomagnesemia on clinical symptoms and adverse events.

Recently published analyses recommend routine magnesium measurements for all patients treated with cetuximab [19]. In the present study, it is shown that specifically patients treated with cetuximab plus platinum derivatives should be monitored for clinical symptoms of hypomagnesemia. The reason for the more severe hypomagnesemia in the platinum plus cetuximab-treated cohort may be platinum-induced nephrotoxicity, which is more severe with cisplatin [22], but has also been reported in oxaliplatin-treated patients [23]. If unusual fatigue or muscle weakness or cramps occur and magnesium levels are low, a discontinuation of cetuximab and intravenous magnesium substitution should be considered [7].

As mentioned before, the predictive value of the early decrease in magnesium serum levels is currently under discussion [12,13]. Using ROC analysis, this analysis showed that a decrease in magnesium levels lower than 95\% of baseline on day 14 of cetuximab exposure is associated with prolonged survival times in mCRC patients. Although data of Vincenzi et al. [12] could be confirmed, the results are in contrast to the report by Vickers et al. [13]. This may in part be explained by the different inclusion criteria and regimens used in the studies. In the present analysis, first-line and second-line patients were treated with cetuximab in combination with chemotherapy, which is similar to the more homogeneous group of Vincenzi et al. [12], where patients 
were treated with irinotecan and cetuximab as the thirdline treatment. However, patients in the report by Vickers et al. [13] were heavily pretreated and received cetuximab as a monotherapy. Detailed data on patients receiving first-line therapy are still missing. A prospective clinical trial with a closer follow-up of magnesium serum levels to validate the retrospective analyses is clearly required to obtain more information on this issue.

\section{Conclusion}

Hypocalcemia is a rare event in cetuximab-treated patients and has no predictive value for efficacy. Patients with impaired renal function as indicated by serum creatinine are more prone to develop hypocalcemia. Therefore, patients with a serum creatinine in the high normal range should be monitored for hypocalcemia when treated with cetuximab. Hypomagnesemia is a common event in cetuximab-treated patients. Data from clinical trials not requiring a close follow-up of magnesium levels probably underestimate the frequency of hypomagnesemia. Because of a greater magnesium waste in patients receiving cetuximab in combination with platinum derivatives, a higher awareness of hypomagnesemia is recommended. If clinical symptoms appear, intravenous magnesium administration and discontinuation of cetuximab are most efficient to restore magnesium levels.

\section{Acknowledgements}

S. Stintzing currently has a postdoctoral fellowship of the German Cancer Aid.

\section{Conflicts of interest}

S. Stintzing has received honoraria for talks and advisory board from Merck-Serono, Amgen GmbH, and Roche AG. D.P. Modest has received honoraria for talks and advisory boards from Merck-Serono, Amgen $\mathrm{GmbH}$, and Roche AG. V. Heinemann has received honoraria for talks and advisory boards from Merck-Serono, Amgen GmbH, and Roche AG. For the remaining authors there are no conflicts of interest.

\section{References}

1 Van Cutsem E, Kohne CH, Hitre E, Zaluski J, Chang Chien CR, Makhson A et al. Cetuximab and chemotherapy as initial treatment for metastatic colorectal cancer. N Engl J Med 2009; 360:1408-1417.

2 Bokemeyer C, Bondarenko I, Makhson A, Hartmann JT, Aparicio J, de Braud F, et al. Fluorouracil, leucovorin, and oxaliplatin with and without cetuximab in the first-line treatment of metastatic colorectal cancer. $J \mathrm{Clin}$ Oncol 2009; 27:663-671.

3 Cunningham D, Humblet $Y$, Siena S, Khayat D, Bleiberg $H$, Santoro A, et al. Cetuximab monotherapy and cetuximab plus irinotecan in irinotecanrefractory metastatic colorectal cancer. N Engl J Med 2004; 351:337-345

4 Bonner JA, Harari PM, Giralt J, Cohen RB, Jones CU, Sur RK, et al. Radiotherapy plus cetuximab for locoregionally advanced head and neck cancer: 5-year survival data from a phase 3 randomised trial, and relation between cetuximab-induced rash and survival. Lancet Oncol 2010; 11:21-28.
5 Lordick F, Luber B, Lorenzen S, Hegewisch-Becker S, Folprecht G, Woll E, et al. Cetuximab plus oxaliplatin/leucovorin/5-fluorouracil in first-line metastatic gastric cancer: a phase II study of the Arbeitsgemeinschaft Internistische Onkologie (AIO). Br J Cancer 2010; 102:500-505.

6 Pirker R, Pereira JR, Szczesna A, von Pawel J, Krzakowski M, Ramlau R, et al. Cetuximab plus chemotherapy in patients with advanced non-small-cell lung cancer (FLEX): an open-label randomised phase III trial. Lancet 2009; 373:1525-1531.

7 Schrag D, Chung KY, Flombaum C, Saltz L. Cetuximab therapy and symptomatic hypomagnesemia. J Natl Cancer Inst 2005; 97:1221-1224.

8 Petrelli F, Borgonovo K, Cabiddu M, Ghilardi M, Barni S. Risk of anti-EGFR monoclonal antibody-related hypomagnesemia: systematic review and pooled analysis of randomized studies. Expert Opin Drug Saf 2012; 11 (Suppl 1):S9-S19.

9 Chubanov V, Gudermann T, Schlingmann KP. Essential role for TRPM6 in epithelial magnesium transport and body magnesium homeostasis. Pflugers Arch 2005; 451:228-234.

10 Ikari A, Okude C, Sawada H, Yamazaki Y, Sugatani J, Miwa M. TRPM6 expression and cell proliferation are up-regulated by phosphorylation of ERK1/2 in renal epithelial cells. Biochem Biophys Res Commun 2008; 369:1129-1133.

11 Stintzing S, Kapaun C, Laubender RP, Jung A, Neumann J, Modest DP, et al. Prognostic value of cetuximab-related skin toxicity in metastatic colorectal cancer patients and its correlation with parameters of the epidermal growth factor receptor signal transduction pathway: results from a randomized trial of the GERMAN AIO CRC Study Group. Int J Cancer 2013; 132:236-245.

12 Vincenzi B, Galluzzo S, Santini D, Rocci L, Loupakis F, Correale P, et al. Early magnesium modifications as a surrogate marker of efficacy of cetuximabbased anticancer treatment in KRAS wild-type advanced colorectal cancer patients. Ann Oncol 2011; 22:1141-1146.

13 Vickers MM, Karapetis CS, Tu D, O'Callaghan CJ, Price TJ, Tebbutt NC, et al Association of hypomagnesemia with inferior survival in a phase III, randomized study of cetuximab plus best supportive care versus best supportive care alone: NCIC CTG/AGITG CO.17. Ann Oncol 2012; 24:953-960.

14 Tejpar S, Piessevaux H, Claes K, Piront P, Hoenderop JG, Verslype C, et al. Magnesium wasting associated with epidermal-growth-factor receptortargeting antibodies in colorectal cancer: a prospective study. Lancet Oncol 2007; 8:387-394.

15 Groenestege WM, Thebault S, van der Wijst J, van den Berg D, Janssen R, Tejpar S, et al. Impaired basolateral sorting of pro-EGF causes isolated recessive renal hypomagnesemia. J Clin Invest 2007; 117:2260-2267.

16 Melichar B, Kralickova P, Hyspler R, Kalabova H, Cerman J Jr, Holeckova P, et al. Hypomagnesaemia in patients with metastatic colorectal carcinoma treated with cetuximab. Hepatogastroenterology 2012; 59:366-371.

17 Costa A, Tejpar S, Prenen H, Van Cutsem E. Hypomagnesaemia and targeted anti-epidermal growth factor receptor (EGFR) agents. Target Oncol $2011 ; 6: 227-233$.

18 Suh SM, Tashjian AH Jr, Matsuo N, Parkinson DK, Fraser D. Pathogenesis of hypocalcemia in primary hypomagnesemia: normal end-organ responsiveness to parathyroid hormone, impaired parathyroid gland function. J Clin Invest 1973; 52:153-160.

19 Do Pazo-Oubina F, Estefanell-Tejero A, Riu-Viladoms G, Anglada-Martinez H, Molas-Ferrer G, Creus-Baro N. Magnesium monitoring practice in monoclonal anti-epidermal growth factor receptor antibodies therapy. J Clin Pharm Ther 2012; 38:101-103.

20 Moosmann N, von Weikersthal LF, Vehling-Kaiser U, Stauch M, Hass HG, Dietzfelbinger $\mathrm{H}$, et al. Cetuximab plus capecitabine and irinotecan compared with cetuximab plus capecitabine and oxaliplatin as first-line treatment for patients with metastatic colorectal cancer: AIO KRK-0104 a randomized trial of the German AIO CRC study group. J Clin Onco/ 2011; 29:1050-1058.

21 Folprecht G, Gruenberger T, Bechstein WO, Raab HR, Lordick F, Hartmann JT, et al. Tumour response and secondary resectability of colorectal liver metastases following neoadjuvant chemotherapy with cetuximab: the CELIM randomised phase 2 trial. Lancet Oncol 2010; 11:38-47.

22 Pabla N, Dong Z. Cisplatin nephrotoxicity: mechanisms and renoprotective strategies. Kidney Int 2008; 73:994-1007.

23 Kawazoe H, Kawazoe H, Sugishita H, Watanabe S, Tanaka A, Morioka J, et al. Nephrotoxicity induced by repeated cycles of oxaliplatin in a Japanese colorectal cancer patient with moderate renal impairment. Gan To Kagaku Ryoho 2010; 37:1153-1157. 